\title{
Repeticiones, membranas y ritmos. Un encuentro entre Gilles Deleuze y Paul Klee
}

\author{
Sergio Martinez Vilajuana \\ Universidad de Chile
}

Resumen: En este trabajo buscaremos proponer un encuentro entre Gilles Deleuze y Paul Klee. Para esto, en primer lugar, consideraremos el concepto de repetición propuesto por Deleuze en el segundo capítulo de Diferencia y repetición; a través de este, el autor francés buscará dar cuenta de la generación de la experiencia del tiempo y, al mismo tiempo, preguntar por el estatuto de la misma. Luego de haber considerado el rol de la experiencia de la repetición, problematizaremos la imagen de ella misma al considerar orgánicamente lo que experimentaría en el límite de sí; y para esto, introduciremos la noción de membrana. Pues bien, si dicha noción complejiza la desenvoltura de la experiencia de la repetición, propondremos volver a problematizarla a través de una noción de ritmo que encontraremos desarrollada en Paul Klee, lo que implicará preguntar por la relación entre arte y naturaleza desde una perspectiva cosmogenética.

Palabras clave: Gilles Deleuze; Paul Klee; repetición; membrana; ritmo

Abstract: "Repetitions, Membranes and Rhythms: an Encounter Between Gilles Deleuze and Paul Klee". In this work we seek to propose an encounter between Gilles Deleuze and Paul Klee. In the first place, we explore the concept of repetition that appears in the second chapter of Deleuze's Difference and repetition. This concept gives an account of the genesis of the experience of time and allows its author to ask about its status. Once we have considered the role of the experience of repetition, we question the image of itself. That is to say, what experiment in the limit of itself. In order to do that, we introduce the notion of membrane. And if this notion makes problematic the unfold in itself of the experience of repetition, we propose to explore this through the notion of rhythm that we find in the thought of Paul Klee. This would imply to ask about the relation between art and nature from a cosmogenetic perspective.

Keywords: Gilles Deleuze; Paul Klee; repetition; membrane; rhythm 
En el Prefacio a Diferencia y repetición, G. Deleuze arroja la siguiente reflexión: "[1]a índole de nuestra vida moderna es tal que, cuando nos encontramos frente a las repeticiones más mecánicas, más estereotipadas, fuera y en nosotros, no dejamos de extraer de ellas pequeñas diferencias, variantes y modificaciones. A la inversa, repeticiones secretas, disfrazadas y ocultas, animadas por el perpetuo desplazamiento de una diferencia, restituyen en y fuera de nosotros repeticiones puras, mecánicas y estereotipadas"1. Independientemente de cuál sea la naturaleza exacta de la repetición o la diferencia, en este breve ensayo intentaremos una aproximación a ella, pues lo que nos interesa es buscar en el movimiento de dicha naturaleza lo que por "fuera y en nosotros (en nous et hors de nous)" habriamos de intentar concebir. Si una de las características del pensamiento deleuzeano ha sido presentarnos un mundo que rebasa "nuestra" escala, esta característica también ha implicado proponer una suerte de restitución creativa $y$ crítica del acto de pensar que buscaría expresar lo que por en y fuera de nosotros intentariamos imaginar. ¿Qué ocurriría si las artes y la literatura nos expresasen lo que en y fuera de nosotros, al otro lado del espejo, nos fuerza a ser nosotros? ¿Acaso, entre el perpetuo desplazamiento de una diferencia y la repetición más mecánica, no nos hallamos ante la potencia de un ritmo que habriamos de intentar atender? Pues bien, si recordamos ahora que en el tan importante capitulo IV de ¿Qué es la filosofia?, titulado "Geofilosofia”, Deleuze y Guattari plantean que: “[e]1 sujeto y el objeto dan una mala aproximación del pensamiento. Pensar no es un hilo tendido entre un sujeto y un objeto, ni una revolución de uno alrededor de otro. Pensar se hace más bien en la relación entre el territorio y la tierra", podríamos, usando una pregunta en tono nietzscheano (¿quiénes somos nosotros?), plantearnos que el cuestionamiento que llevan a cabo Deleuze y Guattari implica un desplazamiento que hace que "nosotros" nos situemos en la relación entre tierra y territorio. En este sentido, el espacimiento del tiempo, la introducción intensiva del espacio en el tiempo, y viceversa, consistiría en la generación o producción de un ritmo que se diferenciaría de la repetición de sí. Es que al abrirnos a una geografia en la

1 Deleuze, G., Diferencia y repetición, Delpy, M.S. y H. Beccacece, (trads.), Buenos Aires: Amorrortu, 2012, p. 16; Différence et Répétition, Paris: Presses Universitaires de France, 2011, p. 2. En adelante para la mayoría de las obras de Deleuze se indicará, después de su primera aparición a pie de página, el título castellano de la obra y el número de página de la edición castellana, seguido del número de página de la edición francesa entre paréntesis. 
que estaría por pensarse el limite de una vida, no intentariamos sino sumergirnos en un "mundo hormigueante", de "singularidades nómades": ¿cuáles serian estas "repeticiones secretas, disfrazadas y ocultas" que deformarian los espejos en que habitualmente nos vemos? ¿Cómo, sino a través de las artes, nos exponemos a aquellos ritmos que nos deforman? Vayamos más lento.

Ya en las primeras páginas del segundo capítulo de Diferencia y repetición, titulado La repetición para sí-misma (La répétition pour elle-même), Deleuze comienza a concebir la noción de repetición en términos de contracción y distención de instantes que, distinguibles empírica y lógicamente, a primera vista habrian de darnos una imagen de una primera síntesis del tiempo ${ }^{2}$ : la sintesis del presente viviente, que habremos de concebir implicada en la experiencia de la repetición. Ahora bien, lo que Deleuze considera al preguntar por el estatuto de los instantes problematizando la repetición para sí-misma-al tornar problemática la diferencia entre lo empírico y lo lógico desde una perspectiva trascendental ${ }^{3}$ no será sino la repetición en y de la experiencia. Sin embargo, no se trataría ni la de un sujeto ni la de un objeto formalmente constituidos, pues aquella misma, en cuanto repetición, no sería sino un "movimiento retroactivo (mouvement rétroactif) entre ambos limites" 4 . Esto es, si la experiencia que se repite en el tiempo se contrae y distiende diferencialmente, la exposición genética de la misma implicará atender a un movimiento retroactivo que nos daría cuenta de ella misma. Y si en Diferencia y repetición la experiencia del tiempo es producto de una síntesis de instantes, la diferencia, al no estar sino implicada en la repetición, tornaría problemática la exposición lógica de los instantes al concebir que lo que los compone $y$ lo que a aquella compone habriamos de considerarlo,

2 Deleuze, G., Diferencia y repetición, pp. 119-120 (96-97).

3 En torno a la noción de empirismo trascendental en Deleuze, cf. Bryant, L., Difference and givennes, Illinois: Northwestern University Press, 2008; Sauvagnnargues, A., Deleuze, L'empirisme transcendantal, Paris: Presses Universitaires de France, 2009, especialmente capitulos I, II y IV; Montenegro, M., Empirismo trascendental. Génesis y desarrollo de la filosofia de Gilles Deleuze, Bogota: Editorial Universitaria (Serie filosófica-Número 23), 2013, especialmente capítulos II y III; Voss, D., Condition of Thought: Deleuze and Transcendental Ideas, Edinburgh: Edinburgh University Press, 2013. Además, cabe mencionar los artículos de Smith, D. W., "Deleuze's Theory of Sensation: Overcoming the Kantian Duality" en: Patton, P. (ed.), Deleuze: a Critical Reader, Oxford: Blackwell Publishers, pp. 29-56; Lord, B., "Deleuze and Kant" en: Smith D.W. y H. Somers-Hall (eds.), The Cambridge Companion to Deleuze, Cambridge: Cambridge University Press, 2012, pp. 82-102.

4 Ibid., p. 120 (97). 
como veremos, vestida o intensivamente ${ }^{5}$. En este sentido, si la repetición de la experiencia implica problematizar el estatuto de los instantes, al considerar que esta misma comportaría otras dos síntesis del tiempo, es en la repetición de los instantes que habriamos de concebir implicada a la diferencia: el movimiento de una diferencia que se desplazaria. En otras palabras, si preguntamos por la naturaleza temporal de dicho movimiento, habriamos de intentar liberarlo de la extensión que lo explicaría en tanto no nos permitiría contemplar aquella contracción y distención de instantes que caracterizarian a la repetición al tornar a los mismos equivalentes entre si, es decir, a homogeneizarlos. Por ejemplo, al considerarlos numéricamente o en términos de sucesión estos no se tornarian sino equivalentes entre sí. En efecto, es la unidad asignable al movimiento de la repetición la problematizada por Deleuze. Nuestro autor va a reiterar, y quizá no es sino una suerte de mantra, que no se trataría ni de lo uno ni de lo múltiple, sino, más bien, de una multiplicidad ${ }^{6}$. A propósito de esto, H. Bergson, retroactivamente leído ${ }^{7}$, consideraba desde una perspectiva ontológica que la idea de multiplicidad podriamos concebirla o bien extensiva o bien intensivamente ${ }^{8}$. Bergson, ya en el fundamental capitulo De la multiplicidad de los estados de conciencia del Ensayo sobre los datos inmediatos de la conciencia, escribia: "la pura duración bien podría no ser sino una sucesión de cambios cualitativos que se funden, que se penetran, sin contornos precisos; sin tendencia alguna a exteriorizarse unos con relación a otros, sin parentesco alguno con el número: sería la heterogeneidad pura. Pero no insistiremos por el momento en este punto: bástenos el haber mostrado que, desde el momento en que se atribuye la menor homogeneidad a la duración, se introduce subrepticiamente el espacio"9.

\footnotetext{
5 Ver, en torno a la noción de cantidades intensivas en el pensamiento de Deleuze y de su lectura de uno de los principios del entendimiento puro propuesto por Kant en la Crítica de la razón pura, el texto el texto de Rölli, M., "Deleuze on Intensity Differentials and the Being of the Sensible", en: Deleuze Studies, v. III, 1 (2009), pp. 26-53, que expone dicha noción desde una perspectiva humeana-kantiana; y las páginas de Ruiz Stull que, leyendo a Deleuze desde una perspectiva bergsoniana, la expone hondando en la relación de Bergson con Kant, Ruiz Stull, M., Tiempo y experiencia. Variaciones en torno a H. Bergson, Santiago: F.C.E, 2013, pp. 65-73.

$364 \quad 6$ "Que lo uno sea una multiplicidad (como también en este caso lo mostraron Bergson y Husserl) basta para desechar juntas las proposiciones de adjetivos del tipo lo uno-múltiple y lo múltiple uno" (Deleuze, G., Diferencia y repetición, p. 277 (236)). Para el diferendo Husserl / Bergson, ver el texto de Ruiz Stull, "Experiencia y empirismo trascendental", en: Díaz, E. (ed.), Gilles Deleuze y la ciencia, Buenos Aires: Editorial Biblos, 2013, pp. 53-64.

7 Deleuze, G., Le bergsonisme, Paris: Quadrige/PUF, 2004, pp. 42-44.

8 Y creemos que no se excluyen ambas consideraciones.

9 Bergson, H., Ensayo sobre los datos inmediatos de la conciencia., Palacios, J.M. (trad.), Salamanca: Ediciones Sígueme, 1999, p. 79; Essai sur les données immédiates de la conscience, Worms, F y Bouaniche, A. (eds.). Paris: PUF, 2011, p. 77.
} 
M. Ruiz Stull, quien ha propuesto una lectura admirable de Bergson ${ }^{10}$, ha problematizado dicha distinción al considerar que la duración, que se le presentaba a Bergson en un principio como una experiencia meramente psicológica y que posteriormente considerará ontológicamente, nos obligaria también a distinguir al espacio del número que se le adscribiría al tornarse real o no-anulable la heterogeneidad intensiva en el despliegue del mismo (pues no se trataría meramente de la experiencia de la conciencia o de una interioridad). En pocas palabras, si la multiplicidad extensiva es la de los instantes considerados lógica y/o numéricamente, la multiplicidad intensiva no sería sino la de los instantes problematizados al considerar una heterogeneidad (duración) implicada en las contracciones y distenciones de los mismos (ella no sería sino esto). Es decir, lo que complejizaría el estatuto de los instantes mismos no sería sino este carácter a-lógico o a-numérico de lo heterogéneo (extra-proposicional, en su expresión a-simbólica) en cuanto indivisible. Pues bien, si consideramos la repetición de los instantes a través de un movimiento retroactivo, ni subjetivo ni objetivo, lo que distintamente podría expresarse sería una experiencia que, liberada de toda posición o representación que totalice o homogenice su devenir ${ }^{11}$, no habriamos de concebirla sino como la de una diferencia. Dicho de otro modo, que la repetición haga del tiempo una experiencia podria decirnos, al atender a la heterogeneidad que la constituiria, que en una composición o continuidad de instantes, al concebirse esta como la de una multiplicidad intensiva ${ }^{12}$, no se expresa sino en la experiencia de un presente-viviente un pasado constitutivo, el mismo que hace del instante presente "el grado más contraído de todo un pasado que es en sí coexistente" al mismo presente ${ }^{13}$. En pocas palabras: no

\footnotetext{
${ }^{10}$ Para el desarrollo de nuestro trabajo es clave el libro Tiempo y experiencia de Ruiz Stull y estamos en deuda con dicha lectura, en tanto replantea la problemática ontológica atendiendo ni más ni menos a lo que las ciencias de nuestra época han dicho (investigado, explorado y expuesto) en torno a la naturaleza misma de las cosas, proponiendo una concepción del movimiento que nos obliga replantear las condiciones bajo las cuales consideramos a la misma experiencia. Valga esta pequeña nota para saludar este extraordinario libro escrito en nuestra lengua.

${ }^{11}$ Como dirá Deleuze, "De cualquiera manera, según la formulación bergsoniana, el tiempo significa que no todo está dado: el Todo no se da. Lo cual no quiere decir que el todo 'se hace' en otra dimensión que sería precisamente temporal, como lo entiende Bergson, o como lo entienden los dialécticos partidarios de un proceso de totalización; sino que el tiempo, último intérprete, tiene el extraño poder de afirmar simultáneamente trozos que de la misma manera que no forman un todo en el espacio, no formen una unidad por sucesión en el tiempo. El tiempo es exactamente la transversal de todos los espacios posibles, comprendidos espacios de tiempos" (Proust y los signos, Monge, F. (trad.), Barcelona: Editorial Anagrama, 1995, p. 136; Proust et les signes, Paris: Presses Universitaires de France, p.157).

12 Deleuze, G., Diferencia y repetición, pp. 333-334 (286-287) y p. 341 (293-294).

13 Ibid., p. 137 (112).
} 
hay presente puro. El presente de la experiencia es ya pasado, y es en este desdoblamiento que en la repetición se desplaza una diferencia. Como dice Deleuze: "Un organismo dispone de una duración de presentes, de diversas duraciones de presente, según el alcance natural de contracción de sus almas contemplativas... De todos modos, sin duda, el presente aparece como fruto de una contracción, pero referido a dimensiones por entero diferentes. En un caso, el presente es el estado más contraído de instantes o de elementos sucesivos, independientes los unos de los otros en sí. En el otro, el presente designa el grado más contraído de todo un pasado, que es en sí, como totalidad, coexistente... El presente solo es el grado más contraído del pasado que coexiste con él si el pasado coexiste en primer lugar consigo, en una infinidad de grados de distensión y de contracción diversos, en una infinidad de niveles"14.

Si lo intensivo es para la experiencia el término para describir la multiplicidad de grados y niveles que la componen, las distintas duraciones que la implican, la diversidad de tiempos que coexisten en la misma, producto de una repetición que se diferencia en sí misma a medida que va contrayéndose y distendiéndose, es el devenir de aquella misma lo que no puede ser considerado como dado. $\mathrm{Si}$, siguiendo a Deleuze, esto es lo que nos descubriría una segunda sintesis del tiempo -la del puro pasado, la de la memoria- también habriamos de decir que el encuentro con la misma dependería de una tercera síntesis del tiempo en el que el ser del devenir se expresaría, y dicho descubrimiento no sería sino producto de un encuentro. Dicho de otro modo, la repetición es siempre desigual en sí misma y una intensidad, implicación de niveles y grados de duraciones temporales, comportaria concebir que el devenir de una multiplicidad, en la que la memoria de la misma en el presente-viviente no sería sino una repetición de la duración en cuanto que repetición ( $y$ diferencia) ${ }^{15}$, no podría sino descubrirse en una tercera síntesis del tiempo. Pues bien, si, por una parte, la diferencia en la repetición no podría concebirse como una sucesión de instantes homogéneos, sino como una heterogeneidad que recorre y compone los instantes, es, por otra parte, al devenir de aquella al que habría que volver para experimentarla como tal (y quizás en este retorno yazca nuestro problema).

Y, "siguiendo la hipótesis bergsoniana, hay que concebir la repetición desnuda como el envoltorio exterior de la vestida (il faut concevoir la répétition nue comme l'enveloppe extérieure de la vêtue): es decir, la repetición sucesiva de los instantes como el más distendido de los niveles coexistentes, (esto es

${ }^{14}$ Ibid., p. 129 y 137 (105 y 112).

${ }^{15}$ Ibid., pp. 356-359 (306-309). 
agregado nuestro), la materia como el sueño o como el pasado más distendido del espiritu"16. Al atender la experiencia de la repetición, lo que una sintesis del tiempo haría de ella no determinaría sino la experiencia misma (como hemos dicho, las contracciones y distenciones son distintas, pues se trata de diversas duraciones temporales). Y si esta fuese la de un organismo o un cuerpo que habriamos de concebir en su composición o bien, en los términos de G. Simondon, en su individuación, soñarla, en el decir de la Ciencia jovial de Nietzsche, implicaría aventurar una incorporación (Einverleibung) de ella misma ${ }^{17}$. Como diría Deleuze, "[s]omos agua, tierra, luz y aire contraidos, no solo antes de reconocerlos o de representarlos, sino antes de sentirlos. Todo organismo es, en sus elementos receptivos y perceptivos, pero también en sus visceras, una suma de contracciones, de retenciones y de esperas"18. Ahora bien, al explorar en la memoria cuando se abre el pasado que es la misma y, como hemos visto, al mismo tiempo, no hay presente sin pasado, ya que la repetición sucesiva de los instantes, no expresa sino los últimos envoltorios de una experiencia compuesta por una infinidad de grados de contracción y distención de presentes-pasados cuya condición no es sino un pasado virtual ${ }^{19}$, algo le ha ocurrido, en esa exposición hacia afuera, a nuestra concepción de la repetición. No podríamos concebir aquel movimiento retroactivo sin considerar que el tiempo implica, para quien hace la experiencia, una heterogeneidad constitutiva. Y si estamos considerando que quien hace la experiencia intensiva de un organismo o un cuerpo no experimenta sino una heterogeneidad constitutiva al mismo, también no sería sino un corte en aquella experiencia el que ocasionaría la posibilidad de volver a experimentarla, o sea, se re-crearía a través de la repetición una diferencia (volveremos sobre esto). Pues, como dirá Deleuze, "una cantidad intensiva se divide, pero no se divide sin cambiar de naturaleza. En un sentido, por lo tanto, es indivisible; pero solo porque ninguna parte preexiste a la división, ni conserva la misma naturaleza al dividirse"20. Que esta división comporte un corte en y de la naturaleza de la intensidad y que, al mismo tiempo, esté implicada en la repetición de la misma, no tiene

\footnotetext{
16 Ibid., p. 139 (114). Cursivas nuestras.

17 Nietzsche, F., La ciencia jovial, Jara, J. (trad.), Caracas: Monte Ávila Editores, 1999, §110.

18 Deleuze, G., Diferencia y repetición., p. 123 (99). Cursivas nuestras.

19 "Pero la memoria trascendental capta lo que, la primera vez, desde la primera vez, solo puede ser recordado: no un pasado contingente, sino el ser del pasado como tal, y el pasado de todo tiempo. Olvidada, de ese modo aparece la cosa en persona a la memoria que la aprehende esencialmente. No se dirige a la memoria sin dirigirse al olvido en la memoria" (Ibid., p. 217 (183)).

20 Ibid., p. 355 (306)
} 
sino relación con quien hace esta experiencia. Y en este hacer de la repetición estaría en juego lo que Deleuze concebirá por tercera síntesis del tiempo.

Pues bien, si dicho corte está formalmente inscrito en la experiencia de la repetición, no será sino a través de un rodeo que intentaremos concebir el mismo. Para esto, comenzaremos dirigiéndonos a una de las lecturas de Kant propuesta por Deleuze ${ }^{21}$. Citamos en extenso: "Toda la crítica kantiana se reduce a objetar contra Descartes que es imposible referir directamente la determinación sobre lo indeterminado. La determinación "pienso" implica evidentemente algo indeterminado ("soy"), pero nada nos dice todavía cómo este indeterminado es determinable por el pienso...La respuesta de Kant es célebre: la forma bajo la cual la existencia indeterminada es determinable por el Yo $(\mathrm{Je})$ pienso es la forma del tiempo. Sus consecuencias son extremas, mi existencia indeterminada no puede ser determinada más que en el tiempo, como la existencia de un fenómeno, de un sujeto fenomenal, pasivo o receptivo que aparece en el tiempo...De un extremo al otro, el YO $(\mathrm{Je})$ se halla atravesado por una fisura: está fisurado por la forma pura y vacía del tiempo. Bajo esta forma, él es el correlato del yo $(m o i)$ pasivo que aparece en el tiempo...Una falla o una fisura en el Yo (Je), una pasividad en el yo $(\mathrm{mol})$, he ahí lo que significa el tiempo; y la correlación entre el yo ( $\mathrm{mo}$ ) pasivo y el yo ( $\mathrm{Je}$ ) fisurado constituye en descubrimiento de lo trascendental o el elemento de la revolución copernicana"22.

Según Deleuze, con Kant el orden del tiempo se libera de determinaciones empíricas y dinámicas: el pasado y el futuro no son sino caracteres fijos y estáticos. Así, lo que definirá el orden del tiempo "como esta distribución puramente formal de lo desigual en sí (el antes y el después, el pasado y el futuro) en función de una cesura" ${ }^{23}$ será el carácter estrictamente formal que caracterizará a la tercera síntesis del tiempo, es decir, a la síntesis estática del tiempo Como dice Deleuze, esta es "forzosamente estática, puesto que el tiempo ya no está subordinado al movimiento (en cuanto sucesión o desplazamiento);

${ }^{21} \mathrm{Cf}$. Deleuze, G., "Sobre cuatro fórmulas poéticas que podrian resumir la filosofia kantiana" en: Crítica y Clínica, Kauf, T. (trad.), Barcelona: Anagrama, 1996, pp. 44-55; "Sur quatre formules poétiques qui pourraient résumer la philosophie kantienne" en: Critique et Clinique, Paris: Les Éditions de Minuit, 1993, pp. 40-49.

${ }^{22}$ Ibid., pp. $141-142$ y 146 (116-117 y 120).

${ }^{23}$ Ibid., pp. 145-146 (120). 
forma del cambio más radical, pero la forma del cambio no cambia"24. Pues bien, si "la cesura es exactamente el punto de nacimiento de la fisura (del sujeto)"25, habremos de decir que la naturaleza de lo que se repite no implica por sí misma "ni la forma subjetiva del pensamiento (Je) ni la de la existencia (Mol)"26; y si bien es un movimiento lo que las actualiza, lo que expresaría la forma del tiempo sería que aquel movimiento no es sino el de una extrema informalidad. Si es una cesura la que se expresa en el orden del tiempo, lo que se repite se experimenta por una fisura en el sujeto pensante, es decir, lo que expone el pensamiento es "un Otro en él" 27.

En pocas palabras, al fisurarse, la experiencia del pensamiento pasa por un cambio en la naturaleza del mismo (y esto se debería a la introducción del tiempo, a la consideración del mismo). Dicho de otro modo, lo que problematizaría las formas subjetivas bajo las cuales el pensamiento y lo existente son figurados para dar cuenta de la hetero-génesis de los mismos estaria formalmente precedido por "una imagen simbólica del tiempo en la medida que reúne una cesura, un antes y un después (en cuanto es a través del tiempo que se aventuraría dicha inasimilable heterogeneidad)"28. Si la experiencia de la repetición en cuanto diferencia "no implica por sí misma" ni la forma subjetiva del pensamiento (Je) ni la de la existencia (Moi), ya que, más bien, "los produce al actualizarse, al efectuarse"29, es porque, como dirá Deleuze, "la extrema formalidad (del tiempo) no está allí más que para un excesivo informal (Unförmliche)"30 que no podría sino aventurarse a través de la experiencia de la tercera síntesis del tiempo. Intentemos invertir los términos, si el "Je" y el "Mol", son formas cuya actualización son producto de un acontecimiento, lo que podría estar en juego en el acontecimiento de una cesura, en una repetición de la diferencia, quizá podría retrotraerse a un organismo liberado de las determinaciones subjetivas solo si este expresase el límite de lo vivo, lo cual sería el caso de una membrana ${ }^{31}$. Vayamos más lento. Deleuze, al introducir este caso en la XV serie de Lógica del

24 Ibid., p. 146 (120). Paréntesis propios.

25 Ibid.

${ }^{26}$ Deleuze, G., Lógica del sentido, (texto) Morey, M. y (apéndice) V. Molina (trad.), Víctor, Madrid: Paidós, 2011, 136; Logique du sens, Paris: Les Éditions de Minuit, 1982, p. 125.

${ }^{27}$ Deleuze, G., Diferencia y repetición., p. 142 (117).

28 Ibid., p. 146 (p. 120). Paréntesis propios.

29 Deleuze, G., Lógica del sentido., p. 136 (125)

30 Deleuze, G., Diferencia y repetición., p. 149 (122-123). Paréntesis nuestro.

31 Deleuze, G., Lógica del sentido, p. 136 (126). 
sentido, serie en la que expresa la idea de un campo trascendental ${ }^{32}$, campo que no habriamos de concebir equivalente al de una conciencia porque esta "no es nada sin síntesis de unificación y no hay síntesis de unificación sin forma del Yo (Je) ni punto de vista del Yo (Mot)"33, implicará el acontecimiento de la cesura en un desarreglo o desasimiento de la misma subjetividad al considerar la génesis de la misma "pre-subjetiva, pre-individual, a-conceptualmente" 34 . Y si "lo que no es ni individual ni personal son las emisiones de singularidades que se hacen sobre una superficie inconsciente y poseen un principio móvil de autounificación por distribución nómada"35, el movimiento retroactivo que habriamos de intentar considerar en el tiempo, el cual comportaría la noción de repetición en la experiencia para concebir la hetero-génesis de la misma en el deshacimiento o desarreglo de la subjetividad, no implicaría ni un sujeto ni un objeto formalmente constituido. Al otro lado del espejo no se trataría sino de otras escalas, en el que el deshacimiento de lo humano implicaria descubrir un otro inconsciente: un mundo a-humano; porque "[c]uando se abre el mundo hormigueante de las singularidades anónimas y nómadas, impersonales, preindividuales, pisamos finalmente el campo de lo trascendental" 36 . Pues bien, si distinguiamos entre una repetición desnuda y una repetición vestida, considerando que aquella no era sino "el envoltorio exterior" de esta última, es decir, haciamos del presente de la repetición el grado más distendido de distintas duraciones o intensidades diferenciales, lo que Deleuze buscaría concebir, al introducir la idea de un principio móvil de autounificación, sería lo que aleatoriamente relacionaría una multiplicidad de grados y niveles de intensidades que irian componiendo la experiencia de la repetición. Esta es, pues, la naturaleza de un "punto aleatorio (point aléatoire)"37, el cual no iría sino autounificando aquella multiplicidad que no se dice sino de la diferencia. En este sentido, no podriamos sustraerle a lo que se repite su aleatoriedad constitutiva, es decir, el carácter nomádico de su distribución (el infinito desplazamiento de una diferencia). En otros términos, una multiplicidad, inmanente en términos de virtualidad, en la generación de aquella superficie, no podría ser concebida como sedentariamente distribuida

\footnotetext{
32 Como bien plantea François Zouravichvilli es en esta serie que "el campo trascendental" se asemeja a lo que con posterioridad denominará Deleuze: "plano de inmanencia", cf. Zouravichvilli, F., El vocabulario de Deleuze, Goldstein, V. (trad.), Buenos Aires: Editorial Atuel, 2007, p. 82.

${ }^{33}$ Deleuze, G., Lógica del sentido, p. 135 (124).

34 Ibid., pp. 133-135 (122-124)

35 Ibid., p. 135 (124-125)

36 Ibid., p. 136 (125).

37 Ibid.
} 
bajo las figuras que se le atribuyen a la conciencia o bajo la figura de la misma conciencia en cuanto lo que autounifica el campo trascendental en el que se pliega y despliega un mundo hormigueante no nos sería atendible ni subjetiva ni objetivamente. En una palabra, si se trata de la introducción del azar o del sinsentido (non-sense) en el pensamiento y en la existencia, esto implica concebir un mundo a-humano al cual no podriamos atribuirle finalidad alguna. Pues bien, lo que el orden del tiempo expondría en la cesura que ordena de una vez el antes y el después ${ }^{38}$ habría que intentar concebirlo a través de la afirmación de la diferencia en tanto intensidad; es decir, un movimiento retroactivo no podría prefigurar la experiencia de la repetición en cuanto que el movimiento de la misma afirmaría de sí la diferencia. Ahora bien, si persiste una energía potencial que la regenera, la recompone y hace que la misma sea la de un organismo o de un cuerpo ${ }^{39}$, es a estos a los que no habriamos sino de atender. Como dirá Deleuze, "Todo ocurre en la superficie, en un cristal que no se desarrolla sino por los bordes. Sin duda, no ocurre lo mismo en un organismo; este no cesa de recogerse en un espacio interior, y de expandirse en el espacio exterior, de asimilar y exteriorizar. Pero no por ello las membranas son menos importantes: ellas llevan los potenciales y regeneran las polaridades; ponen en contacto precisamente el espacio interior y el espacio exterior, independientemente de la distancia. El interior y el exterior, lo profundo y lo alto, solo tienen valor biológico gracias a esta superficie topológica de contacto. Asi pues, hay que comprender incluso biológicamente que 'lo más profundo es la piel'. La piel dispone de una energía potencial vital propiamente superficial. Y, así como los acontecimientos no ocupan la superficie, sino que aparecen en ella, la energía superficial no está localizada en la superficie, sino ligada a su formación y reformación"40.

\section{III}

Así como hay que deshacer las formas de la conciencia para concebir un campo trascendental poblado de singularidades pre-individuales, impersonales, al atender a una superficie en formación y reformación debemos considerar la función que cumple una membrana en un organismo ${ }^{41}$. Hemos visto que si

\footnotetext{
${ }^{38}$ Nos remitimos, antes de todo, a la serie XXI de Lógica del sentido titulada "Del acontecimiento".

39 Ibid., p. 136 (125)

40 Ibid. Cursivas propias.

41 Respecto a un tratamiento general de este problema desde una perspectiva biofilosófica, $c f$ : Ansell-Pearson, K., Germinal Life. The Difference and Repetition of Deleuze, London: Routledge, 1999.
} 
la contracción y distención de duraciones temporales hacen de un organismo o un cuerpo la experiencia de una repetición, el movimiento retroactivo al que habríamos de atender implicaría tanto la actividad del presente sobre el pasado como la del pasado en el presente, esto es, la superficie implica un espesor o no es sino profundidad, y viceversa. Ahora bien, Deleuze se remite a Simondon para pensar en la membrana ${ }^{42}$, diciendo que "la individuación vital crece desde el interior y desde el exterior, y todo el contenido del espacio interior está 'topológicamente' en contacto con el contenido del espacio exterior”43. Recordemos que Simondon en Topología y ontogénesis, uno de los subcapitulos de la segunda parte del libro La individuación, apela al carácter metamórfico de dicho contacto. Si ontogenéticamente puede considerarse a la membrana como aquello que hace que "lo viviente sea a cada instante viviente" 44 es porque lo que la caracteriza no es sino su selectividad. La membrana, al separar una región de interioridad de una región de exterioridad ${ }^{45}$, se caracteriza por la polaridad o asimetría que genera entre el pasado y el porvenir, entre el interior y el exterior, al asimilar selectivamente lo que en el presente hace que lo viviente sea a cada instante viviente. Así, si "no existe, en efecto, distancia en topología; toda la masa de materia viviente que está en el espacio interior está activamente presente en el mundo exterior sobre el límite de lo viviente: todos los productos de la individuación pasada están presente sin distancia y sin demora"46. Sin embargo, es el carácter polarizado y asimétrico de la membrana lo que deja pasar "tal cuerpo en sentido centrípeto o centrífugo y oponiéndose al pasaje de tal otro" ${ }^{47}$. Pues bien, si la superficie es lo más profundo, se debe a que la membrana constituye, para lo viviente, el límite de sí mismo y que viva "sobre su límite" significa que al separar dichas regiones, y en relación a ese límite, genera "una dirección hacia el adentro y una dirección hacia el afuera", "[y] [e]n un organismo pluricelular, la existencia del medio interior complica la topología, en el sentido de que hay varias capas de interioridad y

\footnotetext{
${ }^{42}$ Para la relación entre Deleuze y Simondon, $c f$. Bowden, S. "Gilles Deleuze, a Reader of Gilbert Simondon" en: De Boever, A. y otros (eds.), Gilbert Simondon. Being and Technology, Edinburgh: Edinburgh University Press, 2012, pp. 135-153.

${ }^{43}$ Deleuze, G., "Gilbert Simondon: El individuo y su génesis fisico-biológica" en: La Isla Desierta y otros textos. Textos y entrevistas (1953-1974), Pardo, J. L. (trad.), Valencia: Pre-Textos, 2005, p. 118; "Gilbert Simondon: L'individu et sa genèse physico-biologique" en: L'Tle Déserte, Textes et entretiens 1953-1974, Lapoujade, D. (ed.), Paris: Les Éditions de Minuit, 2002 p. 123.

${ }^{44}$ Simondon, G., La individuación. A la luz de las nociones de forma y de información, Ires, P. A. (trad.), Buenos Aires: Editorial Cactus, p. 286.

45 Ibid.

${ }^{46}$ Ibid., p. 289.

${ }^{47}$ Ibid., p. 286.
} 
de exterioridad"48. Como dirá Simondon, "al nivel de la membrana polarizada se enfrentan el pasado interior y el porvenir exterior: este enfrentamiento en la operación de asimilación selectiva es el presente de lo viviente, que está hecho de esta polaridad entre el pasaje y el rechazo, entre sustancias pasadas y sustancias que advienen, presentes una a la otra a través de la operación de individuación.... Topología y cronología coinciden en la individuación de lo viviente. Es solo posteriormente y según las individuaciones psiquica y colectiva que la coincidencia puede ser rota"49.

Si la topología nos permite concebir lo viviente en su mutación, en su metamorfosis, considerando las capas, los niveles, los grados que van componiéndolo, las repeticiones que van diferenciándolo, la no coincidencia cronológica de lo viviente consigo mismo implica, según las individuaciones psíquicas y colectivas, la problematización de una rotura que haria de aquello lo heterogéneo del pensamiento mismo. Si más arriba deciamos que lo que expresaba la forma vacía del tiempo comportaba hacer de la actividad del pensamiento la de un otro en él, el punto en que nacía la fisura del mismo implicaba una cesura que comportaba decir que lo que volvía de aquel no era sino concebible a través del orden mismo del tiempo. Ello sería, según Deleuze, una forma del tiempo forzosamente estática, una forma del cambio más radical que no estaría sino para un excesivo informal (Unförmliche). Ahora bien, si en Lógica del sentido aquel punto se tornaría simbólicamente pensable en el trazado de una línea que hace frontera de una superficie "que se despliega (se déplie) a ambos lados" de la misma ${ }^{50}$, aquel mismo, en Lógica del sentido, será expresión, por un lado, de los cuerpos, de las afecciones y acciones, $y$, por otro lado, expresará los acontecimientos, las proposiciones y los lenguajes ${ }^{51}$. De esta manera, se presentan dos caras, "siempre desiguales en desequilibrio (toujours inégales en déséquilibre)" 52 , de una superficie física y metafisica ${ }^{53}$. Pues bien, Deleuze considerará que en estas dos caras de una superficie física y metafísica despojada de "un Ser soberanamente individuado, de una Forma fuertemente

\footnotetext{
48 Ibid., pp. 286-287

49 Ibid., p. 290. Cursivas nuestras.

50 Deleuze, G., Lógica del sentido, p. 202 (196).

51 "[T]oda la organización del lenguaje presente las tres figuras de la superficie metafisica o trascendental, de la línea incorporal abstracta y el punto descentrado: los efectos de superficie o acontecimientos; en la superficie, la línea del sentido inmanente al acontecimiento; sobre la línea, el punto del sinsentido (non-sense), sinsentido (non-sense) de superficie copresente al sentido" (Ibid., p. 219 (214)).

52 Ibid., p. 202 (195).

${ }^{53}$ Ibid., p. 158-159 (149-150).
} 
personalizada" 54 , se abriría "un mundo hormigueante de unas singularidades anónimas y nómadas" 55 , de "puntos llamados sensibles"56. En otros términos, la experiencia del pensamiento al aventurarse en aquel excesivo informal (Unförmliche) que la compondría, es decir, en aquel "mundo hormigueante" que compone el plano de la misma, habriamos de intentar concebirla a través de una repetición de la diferencia misma. ¿Quién hace de la repetición una diferencia? ¿Cómo abrir o experimentar ese mundo hormigueante de singularidades? ¿Cuál encuentro con dicha heterogeneidad, con lo que está fuera y en nosotros?

Es al considerar que la membrana constituye para lo viviente el límite de sí mismo, que este caso nos ayuda a proyectar una imagen de lo que deshacería el carácter subjetivo del pensamiento y de la existencia en la tercera síntesis del tiempo. Pues bien, si la cesura concebida en cuanto acontecimiento haría de la repetición de la experiencia misma -de lo que la diferenciaría y la afirmaría-, un límite sobre el que se expondría al excesivo informal que la caracterizaría, es al hacer de la membrana una imagen en la que advendría el tiempo que lo que se repetiría del mismo se habría de intentar atender en cuanto diferencia. Como dirá Deleuze en la Imagen-Tiempo: "La pantalla misma es la membrana cerebral donde se enfrentan inmediatamente el pasado y el futuro, lo interior y lo exterior, sin distancia asignable, independientemente de cualquier punto fijo...Las primeras características de la imagen ya no son el espacio y el movimiento, sino la topología y el tiempo"57.

Ahora bien, en los términos de Simondon, la no coincidencia entre topología y cronología expresaría que la experiencia se diferencia por aquello que no siendo del orden del símbolo se presenta simbólicamente. En este sentido, si la cesura, y el antes y el después que ordena simbólicamente el tiempo, se inscriben formalmente en la experiencia de la repetición, lo que habría de concebirse a través de una membrana en la que justamente se actualizaría aquella experiencia en el límite de sí no sería sino la diferencia. Esto es, a través de aquella misma habría de hacerse una diferencia. Y si esto no fuese más que una suerte de metamorfosis de la naturaleza al arte que sufriría una membrana al no concebirla sino como una imagen en la que adviene un pensamiento que se enfrenta al límite de sí, es decir, a una deformación de su naturaleza, lo que

${ }^{54}$ Ibid., p. 139 (128-129).

${ }^{55}$ Ibid., p. 136 (125).

${ }^{56}$ Ibid., p. 83 (67).

${ }^{57}$ Deleuze, G., La imagen-tiempo, Agoff, I. (trad.) , Barcelona: Paidós, 2015, p. 170; L’imagetemps, Paris: Les Éditions de Minuit, 1985, p. 164. 
estaría en juego sería lo que se haría con "la membrana cerebral". Demos una vuelta a lo que hemos intentado exponer citando extensamente a Deleuze: "E1 Hábito es la sintesis originaria del tiempo, que constituye la vida del presente que pasa; la Memoria es la sintesis fundamental del tiempo, que constituye el ser del pasado (lo que hace pasar el presente)...La primera sintesis, la del hábito, constituía el tiempo como un presente vivo, en una fundación pasiva de la que dependian el pasado y el futuro. La segunda sintesis, la de la memoria, constituía el tiempo como un pasado puro, desde el punto de vista de un fundamento que hacía pasar el presente y advenir otro. Pero en la tercera sintesis [la sintesis estática del tiempo, la del orden del tiempo], el presente no es más que un actor, un autor, un agente destinado a borrarse, y el pasado no es más que una condición que opera por defecto. La sintesis del tiempo constituye aqui un porvenir que afirma a la vez el carácter incondicional del producto con respecto a su condición, la independencia de la obra con respecto a su autor o actor... Hacer de la repetición, no aquello de lo cual 'se sonsaca' una diferencia, ni lo que comprende la diferencia como variante, sino hacer de ella el pensamiento y la producción de 'lo absolutamente diferente'; hacer que, por sí misma, la repetición sea la diferencia en sí misma (faire que, pour elle-même, la répétition soit la différence en elle-même)" 58 .

Lo que quizá estaría buscando Deleuze sería situarnos en una experiencia que, para hacer de sí la diferencia, y en la que el empirismo advendría trascendentalmente, $y$ viceversa, solo podría concebirse artísticamente bajo una acepción nietzscheana ${ }^{59}$. Y si, en el vocabulario de Diferencia y repetición, las síntesis equivalen a las experiencias del tiempo que van produciendo la repetición de la diferencia, la de un hábito cuyo fundamento radica en la memoria, el hacer del porvenir del movimiento de la repetición un objeto o una obra en la que se produce y se piensa lo absolutamente diferente significaría concebirla no solo desprendida de un autor sino también hacer de la obra misma una diferencia.

\footnotetext{
${ }^{58}$ Deleuze, G., Diferencia y repetición, pp. 133 y 151-52 (109 y 125-126)). Cursivas nuestras.

$59 \mathrm{Al}$ menos en la lectura de Deleuze, "L’art précisément invente des mensonges qui élévent le faux à cette plus haute puissance affirmative, il fait de la volonté de tromper quelque chose qui s'affirme dans la puissance du faux. Apparence, pour l'artiste, ne signifie plus la négation du réel dans ce monde, mais cette sélection, cette correction, ce redoublement, cette afirmation. Alors vérité prend peut-être une nouvelle signification. Vérité est apparence. Vérité signifie effectuation de la puissance, élévation à la plus haute puissance. Chez Nietzsche, nous les artiste = nous les chercheurs de connaissance ou de vérité = nous les inventeurs de nouvelles possibilités de vie" (Deleuze, G., Nietzsche et la philosophie, Paris: Presses Universitaires de France, 1983, p. 117).
} 
Pues bien, si lo que se tornaría in-sensible $y$ haría advenir la experiencia de una multiplicidad intensiva, de una duración, de una heterogeneidad, no sería sino la alteración de la experiencia de la repetición misma ${ }^{60}$, una obra de arte o una práctica artística que, en la consigna kleeneana-deleuzeana, hace visibles, audibiles o legibles, fuerzas que en realidad no lo son ${ }^{61}$, podria hacernos experimentar una distorsión o deformación de los sentidos orgánicamente constituidos. Y si consideramos que estas fuerzas, componentes intensivos de la experiencia, pueden hacerse artísticamente visibles, audibles, legibles en dicha alteración para ser experimentadas, es la noción de ritmo elaborada por Paul Klee la que, para nosotros, implicará decir que el límite de lo vivo, una repetición de la diferencia, no advendría sino rítmicamente ${ }^{62}$.

En otras palabras, aquel corte en la afirmación de la diferencia, aquella cesura, dicho acontecimiento en su contra-efectuación, no haría sino repetir en la experiencia de la repetición un ritmo. Paul Klee, en su curso de invierno del año 1922 en la Bauhaus, dedicó tres extraordinarios seminarios teóricoprácticos para explorar dicha noción. En estos propuso una concepción del ritmo expresada formal, gráfica y pictóricamente que hacía de él una sensación en la que se exponía una relación entre arte y naturaleza ${ }^{63}$. Klee, al comenzar dichas lecciones, recuerda un contraste ya introducido en los cursos anteriores que caracterizará bajo los términos de composición y estructura ${ }^{64}$. Esta distinción es fundamental para atender a la noción de ritmo que está en juego tanto en su pensamiento como en su práctica artística. El término "estructura" es útil

${ }^{60}$ Ruiz-Stull, M., Tiempo y experiencia., pp. 237-240

61 Deleuze, G., Francis Bacon. Lógica de la sensación, Herrera, I. (trad.), Madrid: Arena 2009, p. 63; Francis Bacon. Logique de la sensation, Paris: Éditions du Seuil, 2002, p. 57.

62 Habremos de recordar que ya en la primera lección del curso sobre el concepto de diagrama, Deleuze introduce a Paul Klee como uno de aquellos autores-pintores que problematizan de manera notable el problema de la pintura en términos de génesis, caos y ritmo: "eso es interesante para mi pregunta: ¿los pintores pueden aportar conceptos? Sí. [Klee] comienza por decirnos: si ustedes toman en serio la idea de caos, ven que se trata de un no-concepto. El símbolo de ese no-concepto es el punto... Hay que intentar dejarse llevar por ese texto... Es muy filósofo Klee"; "El comienzo del mundo es su asunto, su asunto directo... La música tiene una relación con el comienzo del mundo. ¿De qué manera? Yo no sé bien. Habría que pensar. En todo caso, se puede mezclar todo" (Deleuze, G., Pintura. El concepto de diagrama, Equipo Cactus (trad.), Buenos Aires: Editorial Cactus, 2014, p. 38 y p. 41).

${ }^{63}$ Y el cerebro no deja de jugar un rol fundamental, ver de Klee, P., Kunst - Lehre, Regel, G., Leipzig: Verlag Philipp Reclam, 1987, pp. 184-188.

64 Ibid., p. 136. 
a Klee para atender al nivel constructivo de una obra o, como dirá más tarde, al "esqueleto"65. Para Klee, una estructura específicamente concebida en términos aritméticos, puede sernos de ayuda para atender al carácter composicional (kompositionellen Charakter) de una obra en cuanto permitiria aproximarnos en principio a lo que caracterizaría a los ritmos estructurales (Strukturale Rhytmen). Ahora bien, Klee considerará que no solo son medidas sino también tonos los que podrian llegar a caracterizar, en términos estructurales, a los ritmos que, al ser específicamente expresables los valores tonales por su peso, pueden considerarse bajo similares características. La diferencia radicará en la elección de los medios de presentación pictórica (der bildnerischen Darstellungsmittel) ${ }^{66}$. Esto es, "La primera estructura se sirve solamente de medidas de 'unidad simple de distancia' y de 'doble unidad de distancia'; la segunda, en cambio, se sirve de los pesos de la luz y la oscuridad. Podría decirse, medida, por un lado, peso, por otro lado. O bien, por un lado una fuerza que incrementa su potencia sin exceder en esfuerzo al simplemente doblar el tiempo que representa la distancia $y$, por otro lado, una fuerza que trabaja para lograr incrementar su potencia doblando su energía" 67.

En términos
de medida

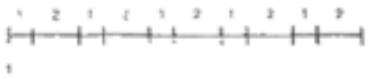

En términos de peso

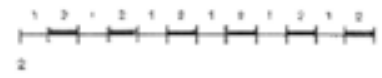

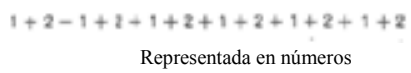

Representada en números

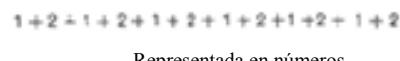

Representada en números

Klee va a complejizar esta concepción estructural del ritmo y, para ello, la diferencia ya expuesta no es menor para atender a esta complejidad, tanto al variar las unidades aritméticas en términos proporcionales como a través de operaciones que diferenciaran a los pesos de las medidas ${ }^{68}$. Ahora bien, para seguir el curso de su exposición, debemos decir que aquella estructura rítmica, considerando dichas variaciones, va conformándose a medida que una unidad rítmica va repitiéndose periódicamente ${ }^{69}$, pero siendo una relación

\footnotetext{
65 Ibid., pp. 180-184.

66 Ibid., p. 137.

67 Ibid., p. 137-138. Traducción nuestra.

68 Ibid., pp. 138-140.

69 Ibid., p. 140.
} 
entre unidades distintas la que determina dicha repetición ${ }^{70}$, dichas unidades pueden hacer variar dicha regularidad ${ }^{71}$. Dicho de otro modo, la repetición periódica (regelmässige Wiederholung) de determinada unidad es una variación de distintos tipos de unidades o de la de una sola unidad rítmica consigo misma, lo cual es clave para atender a la noción de formación estructural (strukturale Formung) que está en juego (fuerzas y energías) ${ }^{72}$. Consideremos lo siguiente: si la consigna kleeneana: "todo devenir yace en movimiento (Bewegung liegt allem Werden zugrunde)"73, enunciada en el Credo creativo (Schöpferische Konfession), se nos torna necesaria de recordar a estas alturas de la exposición, es porque creemos que nos permitirá retornar a Deleuze para volver luego a encontrarnos con Klee. Esto no solo se debe a que para Deleuze no hay oposición entre estructura y génesis, ni entre estructura y acontecimiento o estructura y sentido ${ }^{74}$, sino también porque si entre devenir y movimiento se juega la afirmación de la repetición como diferencia en sí misma, el contraste entre estructura y composición introducido por Klee para pensar en la noción de ritmo buscaría dar cuenta de que es un corte el que da nacimiento a la misma obra. Dicho de otro modo, el ritmo concebido estructuralmente, introducido por un corte y en cuanto corte, nos impulsa, paradójicamente quizá, a considerar una obra en términos de composición: "No puede decirse de tales ritmos que tengan carácter orgánico. Esto es evidente dando un primer vistazo a las series numéricas. Cada organismo es un individuo indivisible. Esto es, no puede quitársele algo sin cambiar su propia naturaleza o, en lo que concierne a los seres vivientes: sin perturbar o impedir el funcionamiento del organismo entero. Del mismo modo, no puede añadírsele algo, cualquiera sea el organismo, sin generar cambios en su naturaleza" 75 .

Si para Klee va a ser necesario ya no hablar en términos de construcción sino de composición ${ }^{76}$, podriamos decir que lo que estaría en juego en la distinción entre devenir y movimiento no podría ser considerado como dado, pues, como dice Deleuze, es necesario hacer de la repetición "el pensamiento

70 Ibid., p. 153.

71 Ibid., pp. 154-156.

72 Klee va a considerar en su curso que el uso de números romanos le permite hacer expresable de que se trata de unidad de mayor complejidad en cuanto implica los distintos niveles (peso y medida) que están en juego en una estructura rítmica. (Ibid., pp. 140-141).

73 Ibid., p. 62.

74 Deleuze, G., Diferencia y repetición, pp. 289 (247).

75 Klee, P., Kunst-Lehre., p. 142.

76 Ibid., p. 41 
y la producción de lo 'absolutamente diferente (l'absolument différent)"'77. Esto es, si lo que está en juego es un asunto de composición, al plantear la naturaleza del movimiento a través de la repetición de unidades rítmicas, el contraste estructural podría generar, como hemos visto, una suerte de movimiento de la diferencia al componer dichas unidades que harian variar lo que a simple vista se nos presentaría como una repetición regular. Utilizando nuevamente el vocabulario deleuzeano, lo que creemos que está en juego en la práctica artística de Klee es la naturaleza de la repetición vestida en cuanto que esta problematiza la noción de ritmo $y$ viceversa ${ }^{78}$. Ahora bien, si la naturaleza de los puntos singulares en Klee es rítmica ¿cómo pensar en el ritmo si este no ha nacido sino del caos ${ }^{79}$ ? Pues bien, si seguimos el planteamiento kleeneano en torno al carácter estructural del ritmo, debemos considerar lo siguiente: "Esta presentación de la estructura rítmica la suponemos expresable en términos cuantitativos (quantitativen Anschauung), es decir, medidas absolutas del tiempo son convertidas en medidas absolutas de longitud (medidas-ritmicas). Pero aquí se revela una deficiencia, que las unidades rítmicas (señalada a través de una barra de compas (Taktstriche)) no pueden ser determinadas con precisión por medio de medidas de longitud. ¿Dónde se sitúa la cadencia $($ Takt)?

\footnotetext{
77 Deleuze, G., Diferencia y repetición., p. 152 (126)

${ }^{78}$ Por supuesto, esto no reduce la complejidad de su obra, pero en este punto no nos podemos extender más. Si atendemos a un período de su obra, habría que concentrarse en un determinado número de obras realizadas entre las décadas de 1920 y 1930. Creemos sin embargo que dicha noción cumple un rol fundamental al considerar las modificaciones composicionales que implican en términos estructurales la variación, bajo consideraciones rítmicas, de líneas y tonalidades, más la dimensión color. Considerando, eso sí, que dichas dimensiones (línea, tonalidades y colores) entran en relaciones distintas en su obra. Más aun, porque si consideramos que el ritmo es una noción entre otras, habría de ser necesario articularla con otras nociones que están también distintamente en juego en su obra, como son, por ejemplo, las de polifonía y simultaneidad (que son propias del periodo que estamos considerando). En torno a la vida y obra de Klee existe un muy buen libro de Hopfengart, C. y M. Baumgartner: Paul Klee. Life and Work, Gallup, A. y otros (trad.), Stuttgart: Hatje Cantz Verlag \& Zentrum Paul Klee Bern, 2012.

${ }^{79}$ Pues como dirá Klee, "[u]n punto en el caos (Ein Punkt im Chaos): el punto-gris establecido, salta sobre él mismo en el dominio ordenado (Der Graupunkt festgestellt, springt ins geordnete Gebiet hinüber) [por el punto gris mismo]". Es que Klee al problematizar en su práctica pictórica un punto que salta del caos y se torna perceptible, hace de él un momento cosmogénetico (kosmogenetische Moment). Pues bien, Klee dirá del caos: "El símbolo para ese 'no-concepto' es el punto matemático, el cual no es realmente un punto. "Algo vacío" o "nada eventual" es un concepto no-conceptual de la falta de oposición. Déjesele tornarse perceptible (como si se extrajese del interior del caos un resultado), llegamos así al concepto de gris, el punto gris: punto fatídico para el devenir y lo que muere" (Klee, P., Das bildnerische Denken, Spiller, J. (ed.). Basilea: Benno Schwabe \& Co - Verlag, 1956, pp. 3-4. Traducción nuestra). Recordemos que Klee cumple un rol preponderante en dicha meseta, ver Deleuze, G. y F. Guattari, Mil mesetas. Capitalismo y esquizofrenia, Vásquez Pérez, J. (trad.) con colaboración de Larraceleta, U., Valencia: Pre-Textos, 2010, pp. 318-319, 382-383, 346-347; Mille Plateaux. Capitalisme et Schizophrénie, Paris: Les Éditions de Minuit, 1980, pp. 382-383, 416-417, 422-423. Traducción nuestra.
} 


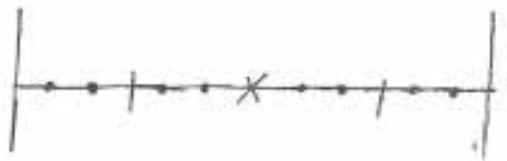

¿Qué significan estos signos específicos como: “”, “.”, "x”, "l”? Ellos indican que bajo ellos, en los lugares en los que se detienen, se introducen diferentes acentuaciones (verschiedene Betonung). Esto nos indica la ruta para pasar a otro tipo de figuración plástica que corresponde de manera más justa y sensible a la naturaleza de la estructura rítmica. Esta presentación ilustraría distintamente la cualidad de dichos signos. Ello consiste en la presentación de las relaciones de peso (Gewichtsverhältnisse), en el tratamiento cualitativo de la estructura rítmica (peso rítmico (Gewischtsrhythmisch))"80.

Ahora bien, Klee considerará que el momento cosmogenético (kosmogenetische Moment) de una obra de arte pictórica es un punto-gris, un punto fatídico para el devenir y lo que muere ${ }^{81}$. Entonces, si atribuirle un tono al gris implicará en él mismo un peso rítmico que comprometerá los demás tonos, la pregunta por el carácter composicional de una estructura rítmica ${ }^{82}$, al atender a las diferentes acentuaciones (verschiedene Betonung) de un peso rítmico (Gewischtsrhythmisch) ${ }^{83}$, no implicaria sino concebir que en la repetición de ese momento cosmogenético (kosmogenetische Moment) se instauraria un ritmo que naceria del $\operatorname{caos}^{84}$. Recordemos que para Klee el arte es una imagen transformada de la naturaleza ${ }^{85}$.

Pues bien, en la implicación de las nociones de repetición, membrana y ritmo en una imagen en la que el tiempo se expresa, habría una cosmogénesis en juego.Y si volvemos a lo dicho en torno a la membrana cerebral en cuanto que imagen ${ }^{86}$, es al caracterizarla topológicamente en su mutación, en su metamorfosis, considerando los niveles y grados de contracción y distención de

${ }^{80}$ Klee, P., Kunst-Lehre, pp. 147-148.

81 Klee, P., Das bildnerische Denken, pp. 3-4.

$380 \quad{ }^{82}$ Uno de los extraordinarios ensayos que propone Klee es el de trasladar, guardando las diferencias, un movimiento a tres voces de Bach a un plano pictórico, al exponer la estructura rítmica que está en juego en dicho movimiento, ver: Klee, P., Kunst-Lehre, pp. 148-151.

${ }^{83}$ Podríamos decir que es por ello que el ritmo se distingue de la cadencia.

${ }^{84}$ Ver nuestras notas a pie número $64 \mathrm{y} 81$.

85 Klee, P., Über die moderne Kunst, pp. 15-17.

${ }^{86}$ Esta imagen es fundamental en ¿Qué es la filosofia? en cuanto propone que los planos filosóficos, científicos y artísticos al luchar distintamente con y contra el caos hagan junción en el cerebro: "la jonction (non pas l'unité) de trois plans, c'est le cerveau" (Deleuze, G. y F. Guattari, ¿Qué es la filosofia?, p. 209 (196)). 
diversas repeticiones que componen la experiencia de un organismo indivisible (espacio-temporalmente), que lo que advendría rítmicamente en una estructura en términos de composición, lo que haria de los ritmos que la componen una afirmación de la diferencia, no sería sino el enfrentamiento contra el caos del cual nacería un ritmo que expondría el límite de una vida. Esto implicaría, quizá, un movimiento de desterritorialización para quien hace la experiencia del tiempo. $\mathrm{Si}$ de un caos, considerando que el caos es aquello que queda fuera de un "un Ser soberanamente individuado, de una Forma fuertemente personalizada" ${ }^{87}$, se ha producido la diferencia, se han compuesto fuerzas de otro modo, un ritmo haría de la génesis misma una duración continua. Pues quizá podría tratarse de un acto de creación que nos haría "rememorar simultáneamente las diferentes partes de esa textura (die verchiedenenen Teile dieses Gefüges gleichzeitig zu vergegenwärtigen)" que puede ser el arte o la naturaleza ${ }^{88}$; un movimiento que al volver sobre sí y, en una pregunta por su relación, no habría sino de afirmar la diferencia en sí misma, producir lo "absolutamente diferente"89. Dicho de otro modo otra relación entre territorio y tierra, una polifónica simultaneidad ${ }^{90}$.

Que sea necesario afirmar dicha diferencia, haciendo de la repetición el pensamiento y la producción de la diferencia en sí misma, es lo que podría estar en juego entre Deleuze y Klee. Dirá Klee: “Ahora, me gustaría considerar la dimensión del objeto figurado en nuevo sentido e intentar mostrar cómo el artista se aproxima a menudo a lo que parece ser una arbitraria 'deformación

87 "F]uera de ese Ser y de esa Forma, no queda sino el caos..." (Deleuze, G., Lógica del sentido., p. 139 (128-129)).

${ }_{88}$ Klee, P., Über die moderne Kunst, pp. 17. Habría que intentar escuchar en la palabra Gefüge el uso que se le da en geología, por tanto estructura pero también textura y tejido.

89 Deleuze, G., Diferencia y repetición., p. 152 (126)

90 Como ha señalado Düchting, H., "Klee traslada este concepto musical en los años 30 hacia el campo de la creación pictórica para poder describir composiciones pictóricas complejas que recíprocamente se interpenetran y complementan formalmente... Lo que Klee comprendía por "pintura polifónica (polyphoner Malerie)" se desprende de sus enseñanzas en la Bauhaus: a través de la superposición de capas distintamente estructuradas se genera una composición de "múltiples voces (Vielstimmigkeit)"; un acorde entre los medios pictóricos, en el que al color se le concede una significación específica" (Klee, P., Malerei und Musik, München: Prestel Verlag, 2001, pp. 5-66. Traducción nuestra)). Como dirá Klee, "Interpenetración como intersección polifónica de diferentes estructuras planas (Flächenstrukturen) y direcciones de movimiento (Bewegungrichtungen)" (Klee, P., Das bildnerische Denken, p. 119. Traducción nuestra)). Nosotros agregamos que Klee ya concibe la polifonía en la composición de líneas, que se generan de puntos (Ibid., p. 103 y 125). La simultaneidad y la polifonía remiten a un ritmo que relaciona ritmicamente dichas capas, y en este sentido los colores cumplen un papel fundamental. 
(Deformation)' de las formas naturales (der natürlichen Erscheinungsform). Ya en principio, él no le concede a estas apariencias naturales el significado coercitivo que sus detractores realistas le atribuyen. Él no se siente tan atado a estas realidades, porque no ve en estas formas terminadas (Form-Ende) la naturaleza del proceso natural de creación (das Wesen des natürlichen Schöpfungsprozesses). Quizás es filósofo, sin querer realmente serlo. Y si este mundo no es el mejor de los mundos posibles como lo declaran los optimistas ni tampoco es tan malo para decir que no sea tomado como un ejemplo, aun así, se dice a sí mismo: ¡En esta figura conformada (ausgeformten Gestalt) no es el único mundo posible! El artista se mueve entonces con una mirada penetrante alrededor de las cosas que la naturaleza le ha formado ante los ojos. Mientras más profundo observe, más fácil le es ensanchar su punto de vista desde el presente hacia el pasado. Más se imprime en él, en lugar de una imagen terminada de la naturaleza (eines fertigen Naturbildes), la imagen esencial de la creación en cuanto génesis (wesentliche Bild der Schöpfung als Genesis). Entonces, también se arriesga a pensar que dificilmente hoy pueda estar ya concluida la creación; de este modo, concibe que aquel acto de creación de mundo (weltschöpferische Tun) se extiende desde atrás hacia delante. Dándole duración a la génesis (Der Genesis Dauer verleihend)" ${ }^{\prime 1}$. Podríamos decir que un ritmo en una obra, enfrentándose con su génesis, haciendo que distintas fuerzas interfieran en su composición, no nos abriría sino el tiempo en y de la obra misma. En la imagen de sí misma, ahí donde una tela (una textura) se ha tornado una membrana cerebral, en el límite de una vida, un movimiento rítmico nacería de un caos para hacer un cosmos.

Recibido: 04/01/2016 Aceptado: 10/10/2016

${ }^{91}$ Klee, P., Über die moderne Kunst, pp. 41-43. Traducción nuestra. 


\section{Bibliografia}

Ansell-Pearson, K., Germinal Life. The Difference and Repetición of Deleuze, Londres: Routledge, 1999.

Baumgartner, M. y C. Hopfengart, Life and Work, Gallup, A., (trad.), Stuttgart: Hatje Cantz Verlag \& Zentrum Paul Klee Bern, 2012.

Bergson, H., Ensayo sobre los datos inmediatos de la conciencia, Palacios, J. (trad.), Salamanca: Ediciones Sígueme, 1999

Bergons, H., Essai sur les données immédiates de la conscience, París: PUF, 2011.

Bowden, S., "Gilles Deleuze, a Reader of Gilbert Simondon", en: De Boever, A., Murray, A., Roffe, J. y A. Woodward, A. (eds.)., Gilbert Simondon. Being and Technology, Edinburgo: Edinburgh University Press, 2012, pp. 135-153. https://doi. org/10.3366/edinburgh/9780748677214.003.0009

Bryant, L., Difference and Givennes., Illinois: Northwestern University Press, 2008.

Deleuze, G., Mille Plateaux. Capitalisme et Schizophrénie, Paris: Les Éditions de Minuit, 1980.

Deleuze, G., Logique du sens, Paris: Les Éditions de Minuit, 1982.

Deleuze, G., Nietzsche et la philosophie, Paris: Presses Universitaires de France, 1983.

Deleuze, G., L'image-temps, Paris: Les Éditions de Minuit, 1985.

Deleuze, G., "Sur quatre formules poétiques qui pourraient résumer la philosophie kantienne", en: Critique et Clinique, Paris: Les Éditions de Minuit, 1993, pp. 40-49.

Deleuze, G., Proust y los signos, Monge, F. (trad.), Barcelona: Editorial Anagrama, 1995.

Deleuze, G., "Sobre cuatro fórmulas poéticas que podrian resumir la filosofia kantiana", en: Crítica y Clínica, Kauf, T. (trad.), Barcelona: Editorial Anagrama, 1996, pp. 44-55.

Deleuze, G., Francis Bacon. Logique de la sensation, Paris: Éditions du Seuil, 2002.

Deleuze, G., L'Tle Déserte, Textes et entretiens 1953-1974, Paris: Les Éditions de Minuit, 2002.

Deleuze, G., Proust et les signes, Paris: Presses Universitaires de France, 2003.

Deleuze, G., Le bergsonisme, Paris: Quadrige /PUF, 2004.

Deleuze, G., La Isla Desierta y otros textos. Textos y entrevistas (1953-1974), Pardo, J. (trad.), Valencia: Pre-Textos, 2005.

Deleuze, G., Francis Bacon. Lógica de la sensación, Herrera, I. (trad.), Madrid: Arena 2009.

Deleuze, G. y F. Guattari, Mil mesetas. Capitalismo y esquizofrenia, Vásquez Pérez, J. (trad.), Valencia: Pre-Textos, 2010.

Deleuze, G., Différence et Répétition, Paris: Presses Universitaires de France, 2011.

Deleuze, G., Lógica del sentido, Morey, M (trad.), Madrid: Paidós, 2011.

Deleuze, G., Diferencia y repetición, Delpy, M. y H. Beccacece (trads.), Buenos Aires: Amorrortu, 2012.

Deleuze, G., Pintura. El concepto de diagrama, Buenos Aires: Editorial Cactus, 2014.

Deleuze, G., La imagen-tiempo, Agoff, I. (trad.), Barcelona: Paidós, 2015.

Deleuze, G. y F. Guattati, Qu'est-ce que la philosophie?, Paris: Les Éditions de Minuit, 2005. 
Deleuze, G. y F. Guattari, ¿Qué es la filosofia?, Kauf, T. (trad.), Barcelona: Editorial Anagrama, 2009.

Düchting, H., Paul Klee. Malerei und Musik, München: Prestel Verlag, 2001.

Klee, P., Das bildnerische Denken, Basel: Benno Schwabe \& Co - Verlag, 1956.

Klee, P., Kunst - Lehre. Leipzig: Verlag Philipp Reclam, 1987.

Klee, P., Teoría del arte moderno, Ires, P. (trad.), Buenos Aires: Cactus, 2008.

Klee, P., Über die moderne Kunst. Berna: Benteli Verlag, 2009.

Lord, B., "Deleuze and Kant", en: Smith, D y H. Somers-Hall (eds.), The Cambridge Companion to Deleuze, Cambridge: Cambridge University Press, 2012. https:// doi.org/10.1017/CCO9780511753657.005

Montenegro, M., Empirismo trascendental. Génesis y desarrollo de la filosofia de Gilles Deleuze, Bogota: Editorial Universitaria (Serie filosófica-Número 23), 2013.

Nietzsche, F., La ciencia jovial, Jara, J. (trad.), Caracas, Monte Ávila Editores, 1999.

Nietzsche, F., La genealogía de la moral, Sánchez Pascual, A. (trad.), Buenos Aires: Alianza Editorial, 2008.

Patton, P. (ed.), Deleuze: a Critical Reader, Oxford: Blackwell Publishers, 1996.

Rölli, M., "Deleuze on Intensity Differentials and the Being of the Sensible", en: Deleuze Studies 3 (1), 2009, pp. 26-53. https://doi.org/10.3366/E1750224109000476

Ruiz Stull, M., Tiempo y experiencia. Variaciones en torno a H. Bergson, Chile: FCE, 2013.

Ruiz Stull, M., "Experiencia y empirismo trascendental”, en: Diaz, E. (ed), Gilles Deleuze y la ciencia, Buenos Aires: Biblos, 2013, pp. 53-64.

Sauvagnnargues, A., Deleuze, L'empirisme transcendantal, Paris: Presses Universitaires de France, 2009.

Simondon, G., La individuación. A la luz de las nociones de forma y de información, Ires, P. (trad.), Buenos Aires: Cactus, 2015.

Voss, D., Condition of Thought: Deleuze and Transcendental Ideas. Edinburgo: Edinburgh University Press, 2013. https://doi.org/10.3366/edinbu rgh/9780748676255.001.0001

Zouravichvilli, F., El vocabulario de Deleuze, Goldstein, V. (trad.), Buenos Aires: Atuel, 2007. 